\title{
Buckling of single-walled carbon nanotubes using two criteria
}

\author{
Shakti S. Gupta, ${ }^{1, a)}$ Pranav Agrawal, ${ }^{1}$ and Romesh C. Batra ${ }^{2}$ \\ ${ }^{1}$ Mechanics and Applied Mathematics Group, Department of Mechanical Engineering, Indian Institute \\ of Technology Kanpur, Kanpur 208016, India \\ ${ }^{2}$ Department of Biomedical Engineering and Mechanics, M/C 0219, Virginia Polytechnic Institute \\ and State University, Blacksburg, Virginia 24061, USA
}

(Received 7 February 2016; accepted 11 June 2016; published online 28 June 2016)

\begin{abstract}
We use molecular mechanics simulations with the MM3 potential to study instabilities in clampedclamped single-walled carbon nanotubes (SWCNTs) deformed in torsion and axial compression. The following are the two criteria employed to find the critical buckling strain: (i) a sudden drop in the potential energy and (ii) an eigenvalue of the mass weighted Hessian of the deformed configuration becoming zero. The instability under axial compression is investigated for zigzag and armchair SWCNTs, and that under torsional deformations is also studied for chiral tubes. In general, values of critical strains from the 2nd criterion are found to be substantially less than those from the 1st criterion. For chiral SWCNTs, the critical strains from the 2 nd criterion and the potential energies at the onset of instability markedly depend upon the twisting direction. Values of buckling strains predicted from the column and the shell buckling theories are found to agree well with those obtained using the 2nd criterion. Published by AIP Publishing.

[http://dx.doi.org/10.1063/1.4954686]
\end{abstract}

\section{INTRODUCTION}

Successful applications of single-walled carbon nanotubes (SWCNTs) as reinforcing fibers in a composite laminate depend on the accurate determination of their elastic constants, usually defined for a continuum. For a SWCNT (a discrete atomic structure), they can be found by hypothesizing that the tube deforms like a continuum, e.g., a beam or a shell or a cylinder. To determine these constants, one either performs molecular mechanics/molecular dynamics (MM/ MD) simulations or conducts experiments on a SWCNT under various static and dynamic deformations, e.g., axial tension/compression, bending, torsion, radial expansion/contraction or combination of these. Then, an equivalent continuum structure (ECS) of the SWCNT is sought whose response matches with that of the SWCNT under identical loading and boundary conditions. This process also involves assumptions regarding the material symmetry group for the ECS, and whether deformations are elastic, inelastic, infinitesimal, or finite. A single layer graphene sheet (SLGS) has a six-fold hexagonal symmetry ${ }^{1}$ in its basal plane. An SWCNT, thought to be formed by rolling an SLGS, is assumed to be either isotropic or transversely isotropic with the axis of transverse isotropy either along tube's axis or a radial line.

One procedure for determining elastic constants of a CNT is to substitute the value of either its axial compressive or torsional buckling strain derived from either MM/MD simulations or physical experiments into the appropriate expression for the ECS. This method can also be used to determine geometric parameters such as the wall thickness of S/MWCNTs. The literature briefly summarized below

\footnotetext{
${ }^{a}$ Author to whom correspondence should be addressed. Electronic mail: ssgupta@iitk.ac.in.
}

suggests that the following two criteria have been used to identify the critical buckling strain:

1. The onset of buckling is characterized by a sudden/sharp drop in either the potential energy $U$ (or axial force) vs. the axial strain, $\varepsilon$, curve or the $U$ (torque) vs. the shear strain, $\gamma$, curve. This criterion is henceforth termed as Criterion-1 in this paper.

2. An SWCNT buckles at the axial/shear strain for which a non-trivial eigenvalue of the Hessian of the strained SWCNT vanishes. This criterion is termed as Criterion-2.

We briefly review below the pertinent literature on buckling of SWCNTs first for Criterion-1 and then for Criterion-2.

Employing the Tersoff-Brenner (TB) potential in MD simulations, Yakobson et $a l^{2}{ }^{2}$ studied axial, bending, and torsional buckling instabilities in armchair and zigzag SWCNTs. During axial compression of $(7,7)$ SWCNTs and torsion of $(13,0)$ SWCNTs with gradually incremented applied loads, they found discontinuities (kinks or sudden drops) in the potential energy, $U$, of the tubes. They associated the critical buckling strain, $\varepsilon_{c r}$, with the first such drop in the strain energy. Employing a thin shell theory, assuming the material of the ECS to be linear elastic and isotropic, ${ }^{1}$ and equating the $\varepsilon_{c r}$ deduced from the MD simulations to that of the ECS, they determined Young's modulus and the wall thickness of SWCNTs to be $5.5 \mathrm{TPa}$, and $0.66 \AA$, respectively. While studying axial compression of a $(8,0)$ SWCNT using a general tight-binding MD potential, Srivastava $e t$ al. ${ }^{3}$ found a sudden drop in $U$ at $12 \%$ axial strain and the corresponding critical axial stress $\sigma_{c r} \approx 153 \mathrm{GPa}$ using Euler's column theory. Similarly, Liew et al. ${ }^{4}$ used the second generation Brenner potential and computed values of critical load $P_{c r}$, for several armchair and zigzag SWCNTs. For $(8,0)$ SWCNTs, they 
found $\sigma_{c r}=149 \mathrm{GPa}$. Srivastava et al. $^{3}$ and Liew et al. ${ }^{4}$ compared their values of the critical stress with those found experimentally by Lourie et al..$^{5}$

Sears and Batra ${ }^{6}$ studied axial compression and torsional buckling of short and long $(16,0)$ SWCNTs using the MM3 potential, identified the critical buckling strains with a sudden drop in $U$, and found that these differed from those determined by using Euler's column and Donnell's shell theories. Other works using this criterion are briefly stated. To study buckling of zigzag and armchair SWCNTs deformed in axial compression, torsion, and bending, Cao and $\mathrm{Chen}^{7}$ used MM simulations. Zhang and Shen ${ }^{8,9}$ analyzed axial and torsional deformations of armchair and zigzag SWCNTs using MD simulations employing the second-generation reactive empirical bond-order (REBO) and the LennardJones (L-J) potentials. Using the condensed-phase optimized molecular potentials for atomistic simulation studies (COMPASS) force field in MD simulations at $1 \mathrm{~K}$, Wang et $a l{ }^{10}$ studied torsional buckling of armchair and zigzag SWCNTs and identified critical angle of twist from a sudden drop in $U$. Using MD simulations with the Morse and the L-J potentials to study axial compressive buckling of perfect and defective armchair and zigzag SWCNTs, Xin et al. ${ }^{11}$ computed the $\varepsilon_{c r}$ and $P_{c r}$. Using two different values of $E$ and $h$ with their product $=\sim 360 \mathrm{~N} / \mathrm{m}$ in Euler's column buckling formula they found good agreement in values of $P_{c r}$ and $\varepsilon_{c r}$ with those obtained in MD simulations for slenderness ratio (length/diameter) $>13$. Zhang et al. ${ }^{12}$ studied dynamic buckling of SWCNTs using the REBO and the L-J potentials, and they found that the axial strain rate increases the $P_{c r}$ and the corresponding axial strain, $\varepsilon_{c r}$. Wang et al. ${ }^{13}$ studied buckling of SWCNTs using MD simulations employing the COMPASS force field and determined $\varepsilon_{c r}$. Next, using formulae for $\varepsilon_{c r}$ in inelastic buckling of shells, and the value of the tangent elastic modulus, $E_{t}$, computed from an elementary combination of stretching and bending terms in potentials of carbon atoms, they showed a good match in $\varepsilon_{c r}$ from a continuum model and MD simulations. Buckling under compression in armchair and zigzag SWCNTs has been investigated by Zhang et al. ${ }^{14}$ using MD simulations employing the REBO and the L-J potentials to study axial buckling of a $(10,0)$ SWCNT. Vijayaraghavan and Wong ${ }^{15}$ studied buckling of initially imperfect SWCNTs. Using the COMPASS force field and the REBO potential, Kuang et $a l .{ }^{16}$ and Garg and Sinnott, ${ }^{17}$ respectively, studied buckling of functionalized SWCNTs under axial compression, and found that the critical buckling strain decreased after functionalization. Wong and Vijayaraghavan ${ }^{18}$ studied buckling of $(10,10)$ SWCNT submerged in water using MD simulations employing the Brenner and the L-J potentials for carbon atoms and flexible fixed-point-charge potential for water molecules. They showed that the submerged SWCNT buckled prematurely as compared to the isolated one.

Using the COMPASS force field in MD simulations, Cao et al. ${ }^{19}$ studied shifts in frequencies of the fundamental modes of transverse vibrations of armchair and zigzag SWCNTs to determine the strain (but not the buckling strain) in tubes pre-deformed in tension, compression, bending, and torsion. Chang et al. ${ }^{20}$ used MM simulation results to derive analytical expressions for buckling of armchair and zigzag SWCNTs deformed in axial compression. They found that their values of the $\varepsilon_{c r}$ are higher than those predicted by the thin shell model employed by Yakobson et al. ${ }^{2}$ Yeak et al. ${ }^{21}$ studied elastic and plastic properties of zigzag and armchair SWCNTs using a multiscale method. They used a semiempirical tight-binding method and the second-generation REBO potentials in the central and the outer regions, respectively. Using the Brenner potential in MD simulations Garg et $a l .^{22}$ studied axial compression of $(10,10)$ SWCNTs of different aspect ratios (length/diameter) against the $\mathrm{C}(111): \mathrm{H}$ surface and an SLGS. A sudden drop from the maxima in the force-deflection curve was recognized as the critical buckling load, $P_{c r}$. Assuming the ECS of a SWCNT to be a solid circular cylinder and the Euler buckling theory, they found Young's modulus to be between 0.8 and 2.3 TPa.

Using the REBO and the L-J potentials, Chang ${ }^{23}$ associated the critical shear strain with a sharp change in the plot of the shear stress vs. the shear strain curve and showed that the torsional buckling strains of chiral SWCNTs depend on the loading direction. From a discontinuity in the force vs. the displacement curve for MWCNTs in a nano-indentation test, Jeng et al. ${ }^{24}$ determined the critical load, $P_{c r}$. Using the Euler column buckling formula and considering a MWCNT to be a hollow cylinder, they computed values of the critical stress and Young's modulus. Jeong et $a{ }^{25}$ have studied buckling of armchair single-, double-, triple-, quadruplewalled nanotubes, a bundle of SWCNTs, and filled (with $\mathrm{C}_{60}$ and $n$-butane) SWCNTs deformed in torsion using MD simulations with the REBO and the L-J potentials, and identified the onset of buckling with a discontinuity in the torque versus the angle-of-twist curve.

Zhang et $a l .{ }^{26}$ studied nonlinear elastic response of infinitely long armchair SWCNTs in torsion using objective MD. In the objective $\mathrm{MD},{ }^{48}$ the potential is simplified by considering the helical symmetry rather than invariance with respect to translations only. The graph of $U$ vs. angle of twist/length $(\phi)$ for a $(12,12)$ SWCNT in their study did not show any discontinuity for values of $\phi$ up to $16^{\circ} / \mathrm{nm}$. However, the value of torque (proportional to the derivative of $U$ with respect to $\phi$ ) abruptly fell at $\phi=4^{\circ} / \mathrm{nm}$ after attaining a peak value. They also found that at $\phi=4^{\circ} / \mathrm{nm}$ the morphology of the SWCNT changed from cylindrical to that of a twisted shell with two circumferential waves. Using symmetry arguments, they ${ }^{26}$ identified the sequence of bifurcation points corresponding to different morphologies and also determined mode shapes corresponding to the lowest energy. For a three-walled CNT, they deduced similar results. Khademolhosseini et al. ${ }^{27}$ developed a nonlocal elasticity theory based shell model to study torsional buckling of SWCNTs of aspect ratios between $\sim 15$ and $\sim 30$. The $U$ vs. the shear strain, $\gamma$, graph for a $(8,0)$ SWCNT obtained from MD simulations employing adaptive intermolecular reactive empirical bond order (AIREBO) potential did not show a sudden drop; however, the torque $(T)$ vs. the $\gamma$ graph derived from energy showed discontinuity at the onset of torsional instability.

Poelma et $a l .{ }^{28}$ have employed the Dreidling force field in MD simulations to study axial buckling of S/MWCNTs. 
A sudden drop in the force vs. the axial strain plot was identified as the occurrence of buckling. Employing the TB potential in MD simulations, Eftekhari et al. ${ }^{29}$ have studied axial buckling of armchair and zigzag S/MWCNTs. From a sudden drop in the force vs. the compressive axial strain curve, the value of the critical strain was determined. For SWCNTs of aspect ratio $>21$, they found that the Euler buckling formula provided good results. For aspect ratio $<21$, their simulations predicted the shell wall buckling mode. Chowdhury et al. ${ }^{30}$ have studied mechanical behavior of joined armchair and zigzag SWCNTs under axial tension and compression, and torsion. The buckling load in compression for these tubes is found from a sudden drop in the loaddeflection curve.

Annin et al. ${ }^{31}$ have modeled $(10,0)$ SWCNTs using mass for $\mathrm{C}$ atoms and truss elements for bonds in the finite element method to study torsional buckling. They considered the Morse potential for modeling the central force between bonded atoms and truss elements to simulate small bending between the bonds. In the quasistatic method, they found the critical value of torque, $T_{c r}$, (the quasi-bifurcation points) by solving the generalized eigenvalue problem by considering the tangent stiffness matrix at each incremental twist. In the second method, they solved equations of motion iteratively using the Newton-Raphson method, and found different morphologies of the SWCNT in the post-buckling regime. Subsequently, Annin et al. ${ }^{32}$ incorporated van der Waals interactions using the L-J potential in their previous work ${ }^{31}$ and studied buckling and postbuckling behaviors of $(10,10)$ and $(10,0)$ SWCNTs.

Silvestre $^{33}$ showed that the Sanders' shell theory together with a non-uniform helically deflected shape provided a better agreement between MD and continuum results for torsional buckling of SWCNTs than the Donnell's shell model.

Kinoshita et al. ${ }^{34}$ have studied axial buckling of pristine and defective SWCNTs (aspect ratio $\sim 20$ and $\sim 42$ ) using MM simulations with the AIREBO potentials. The defects were introduced in SWCNTs to generate symmetric (Sshaped) and asymmetric (C-shaped) initial configurations. The force vs. the compressive axial strain plot for pristine and symmetric initial configuration of SWCNTs showed a distinct bifurcation in the load path indicating the occurrence of buckling. They validated it from vanishing of the eigenvalue of the Hessian for symmetrically defective and short SWCNTs. We note that Shima ${ }^{35}$ has provided an exhaustive review of buckling of CNTs.

The literature briefly reviewed above suggests that Criterion-1 is widely followed and Criterion-2 is sparsely investigated. Moreover, the buckling of the same SWCNTs according to the two criteria using the same MM potential has not been investigated, an exception being Ref. 34 .

We employ here the MM3 potential ${ }^{36}$ and the MM simulations at $0 \mathrm{~K}$ to find critical buckling strains using Criteria 1 and 2 during axial and torsional deformations of armchair and zigzag SWCNTs and only torsional deformations of chiral SWCNTs. For an SWCNT, the results obtained from Criterion-2 are found to significantly differ from those computed by using Criterion-1. In axial compression, SWCNTs are found to buckle either in shell or beam mode depending on their aspect ratios. However, in torsion, the tubes buckle only in shell modes (SM) for the aspect ratios considered.

The rest of the paper is organized as follows. In Section II, details of the MM3 potential and simulations are described. The response of SWCNTs to torsional and axial deformations is discussed in Sections III and IV, respectively. In Section V we compare the critical buckling strains in torsion and axial compression using both the criterion for SWCNTs of various chiralities and aspect ratios. A possible reason for differences in critical strains from the two criteria is given in Section VI. Findings of this work are summarized in Section VII.

\section{DETAILS OF MM SIMULATIONS}

\section{A. MM3 potential}

The MM simulations have been performed with the open source code TINKER. ${ }^{37}$ The interaction between carbon atoms is specified with the MM3 ${ }^{36}$ potential $U$ given by

$$
\begin{aligned}
U= & \sum_{p} \sum_{q}\left(U_{s}+U_{\theta}+U_{\phi}+U_{s \theta}+U_{\phi s}+U_{\theta \theta^{\prime}}\right) \\
& +\sum_{p} \sum_{r} U_{v d W},
\end{aligned}
$$

in which $U_{s}, U_{\theta}$, and $U_{\phi}$ account, respectively, for the changes in the bond length, the angle between adjoining bonds, and the dihedral torsion. $U_{s \theta}$ and $U_{\phi s}$ are potentials due to interactions between stretching and bending deformations, and between stretching and twisting deformations. $U_{\theta \theta^{\prime}}$ represents interactions between different bending modes. $U_{v d W}$ is the potential for the non-bonded van der Waals forces, and its expression involving terms $\left(r_{\nu} / r\right)^{6}$ and $\exp \left(-12 r / r_{\nu}\right)$ are different from that in the L-J potential. In the expression for $U$, the index $q$ ranges over bonded atoms and the index $r$ over all atoms. As listed in Table III of Gupta and Batra, ${ }^{38}$ the MM3 potential gives frequencies of radial breathing modes of SWCNTs that agree well with those experimentally found. ${ }^{39}$ Since no structural model is used in this comparison, the close agreement between the computed and the experimental values provides one measure of the validity of the MM3 potential to model SWCNTs. Another indication of the suitability of the MM3 potential for SWCNTs is that the computed basal plane stiffness of graphene nanoribbons of $340 \mathrm{~N} / \mathrm{m}$ in tensile and free vibration tests ${ }^{40}$ is very close to the experimental mean value of $340 \mathrm{~N} / \mathrm{m}$ found by Lee et al. ${ }^{41}$ Expressions for various terms of the MM3 potential and values of parameters are given, amongst other sources, in Shah and Batra. ${ }^{42}$

\section{B. Procedure}

An SWCNT without constraints on atomic degrees of freedom and with no cut-off distance is first relaxed to find the minimum energy configuration (or the reference configuration) to within $0.001 \mathrm{kcal} / \mathrm{mol} / \AA \mathrm{rms}$. The variation in the potential energy and its gradient with the iteration number in the minimization process for a SWCNT is shown in Fig. 1. Subsequently, the clamped boundary condition is applied to rings of carbon atoms located inwards from the two ends of 


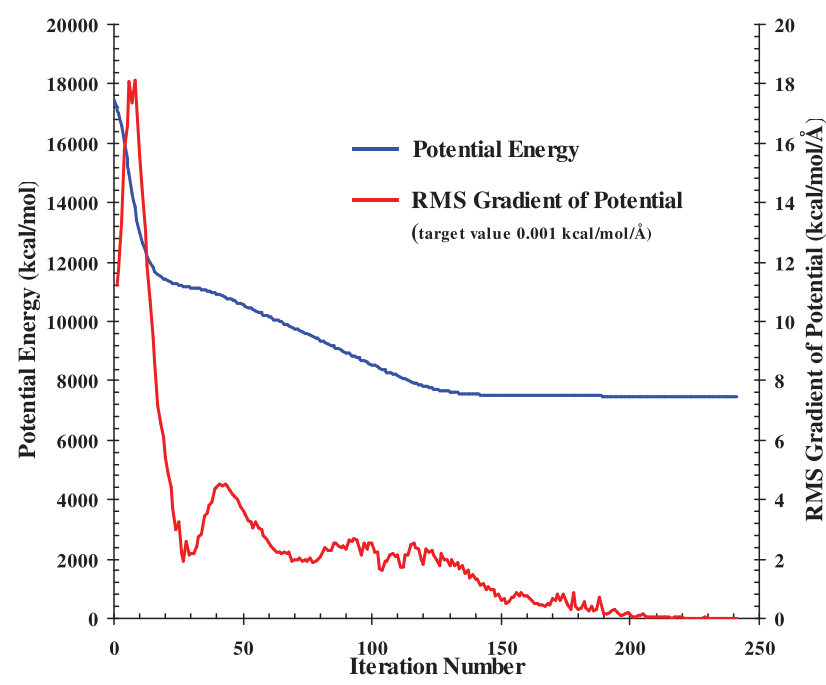

FIG. 1. Variation with the iteration number in the value of the potential energy $U$ and of the RMS value of its gradient during the energy minimization process for unconstrained $(16,0)$ SWCNT. The radius and the length $(R, L)$ in $\AA$ before and after the minimization process for this SWCNT are $(6.264,191.700)$ and $(5.939,181.303)$.

the tube by constraining to zero their displacements $u_{x}, u_{y}$, and $u_{z}$, in the $x$-, the $y$-, and the $z$-directions, respectively. Incremental axial and tangential displacements are then applied to atoms at one end of the tube. Keeping these atoms fixed at the new locations during the relaxation process, the potential energy $U$ of the deformed tube is recorded. The deformation corresponding to the sharp drop in $U$ equals the buckling strain according to Criterion-1.

The buckling Criterion-2 requires computation of eigenvalues (frequencies) and associated eigenvectors (mode shapes) of mass weighted Hessian at each increment of deformation or of the tangent stiffness matrix. This is accomplished by using VIBRATE subroutine in TINKER. In structural mechanics, mode shapes of a cylindrical shell are represented by a pair of real numbers $(i, j)$, where $i$ and $2 j$ are, respectively, the circumferential and the axial wave numbers. Thus, torsional, axial, and radial breathing modes are represented by $(0,1)$, and the beam type bending modes by $(1, j)$. Eigenvalues computed using VIBRATE module have units of wave number in $\mathrm{cm}^{-1}$. This can be converted to $\mathrm{Hz}$ by multiplying it with the speed of light in $\mathrm{cm} / \mathrm{s}$.

We note that results exhibited in different figures correspond to the engineering axial strain and the engineering shear strain. That is, the axial strain equals the change in length per unit undeformed length, and the shear strain the relative tangential or circumferential displacement of the end faces divided by the axial distance between them. These definitions provide reasonably accurate values of strains up to the onset of buckling for both criteria 1 and 2 since crosssections remained circular.

\section{TORSIONAL DEFORMATIONS OF SWCNTS}

We study elastic instability in SWCNTs of three chiralities and of different aspect ratios undergoing torsional deformations.

\section{A. Armchair tube}

We begin with the $(12,12)$ SWCNT of aspect ratio $\sim 12$ shown in Fig. 2(a). Red colored atoms on the front face are kept fixed. Atoms on the far end face are given incremental tangential displacements and then are held fixed to obtain the minimum energy configuration. The variation in eigenvalues $(k)$ for different modes and the total potential energy under incremental shear strain $(\gamma)$ for this SWCNT are shown in Fig. 3.

\section{Buckling according to Criterion-2}

The results depicted in Fig. 3 imply that eigenvalues for the first bending mode (BM) $(1,1)$ and the first torsion mode (TM) $(0,1)$, shown in Figs. 2(a) and 2(b), respectively, do not appreciably change with increasing angle of twist. However, eigenvalues of shell modes (SM) $(2,1),(2,2),(2,3)$, and $(3,1)$ decrease monotonically with an increase in $\gamma$ before vanishing at $\gamma=4.6 \%, 5.3 \%, 6.4 \%$, and $8.2 \%$, respectively. These critical values of $\gamma$ correspond to the onset of instability of
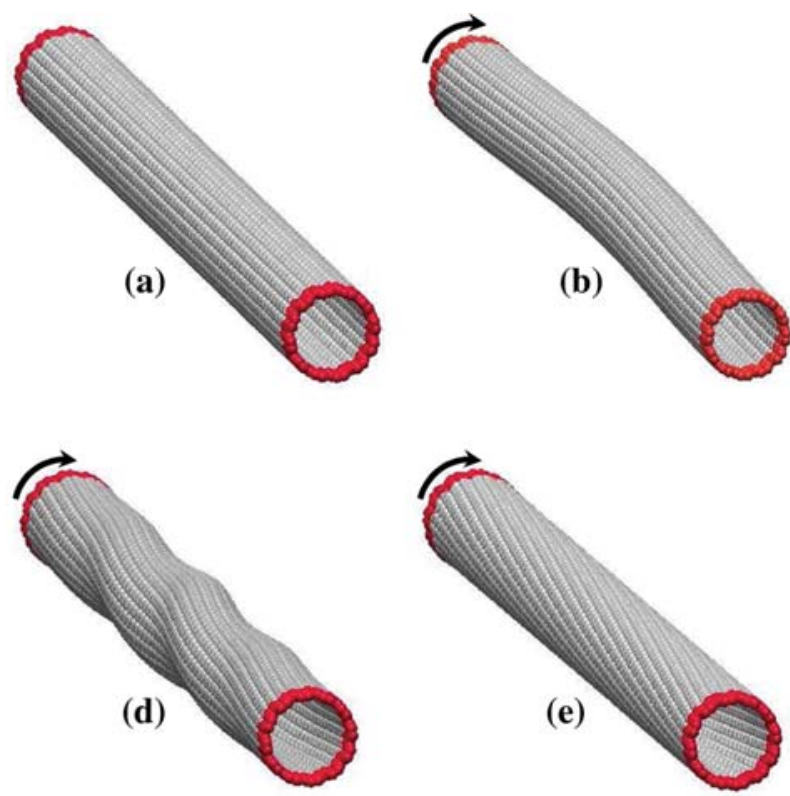
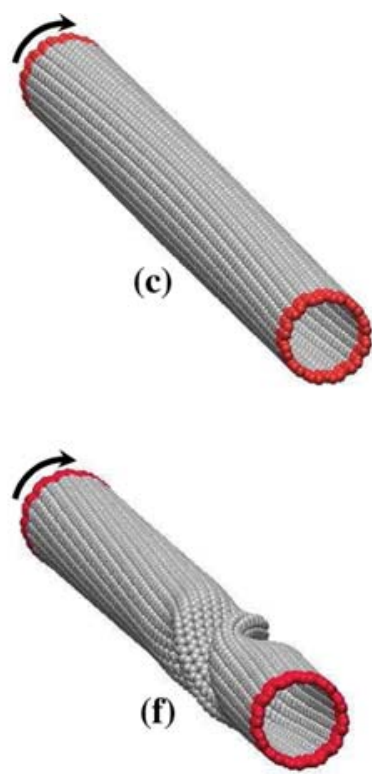

FIG. 2. Various morphologies of $(12,12)$ SWCNT under torsion (the direction of the applied tangential displacement is shown with thick black arrow); (a) the reference configuration, (b), (c) the first bending mode $(1,1)$ and the first torsion mode $(0,1)$ of vibration at $2 \%$ shear strain, (d) the tube at $8.0 \%$ shear strain on the bifurcated potential energy curve $B C$ for $i=2$, (e) the tube at $11.1 \%$ shear strain on the potential energy curve $O B D$, and (f) the collapsed tube with three circumferential waves at $11.2 \%$ shear strain, point $E$, on the potential energy curve. 


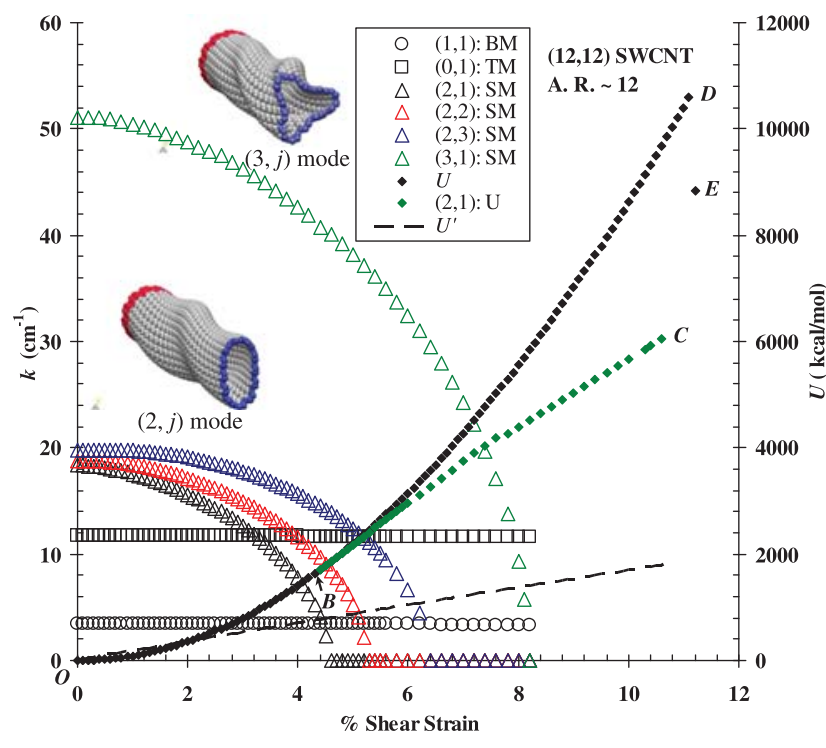

FIG. 3. Variation with shear strain, of the total potential energy $(U)$, eigenvalues $(k)$ of various modes of vibrations (bending, torsion, shell modes $(i$, $j)$ ), bifurcated potential energy corresponding to $(2,1)$ mode, and the slope $U^{\prime}$ of the total potential energy. The shell mode $(2,1)$ bifurcates at point $B$ on curve $O B D . B C$ is the bifurcated path of the shell mode $(2,1)$. Crosssectional views for typical $(2, j)$ and $(3, j)$ vibration modes are also shown in the inset. Curves $B C, O B D$, and point $E$ are depicted in Fig. 3. A. R. stands for aspect ratio (length/diameter).

the respective modes. Cross-sections of the strained SWCNT vibrating in $(2, j)$ and $(3, j)$ modes are shown in the inset in Fig. 3. Furthermore, we note that the critical shear strain at which the eigenvalue of $(2,1)$ mode vanishes is the least. Thus, according to Criterion-2, the tube will buckle under torsion in $(2,1)$ shell mode at $\gamma=4.6 \%$. To test if the tube has indeed buckled in $(2,1)$ mode, we minimized the potential energy of the tube considering $(2,1)$ mode as its reference configuration at shear strains a little less and a little more than $4.6 \%$. This starting configuration was obtained from the VIBRATE subroutine. For shear strains $<4.6 \%$, the morphology of the tube corresponding to the minimum energy was found to be straight and circular, although twisted. However, this did not happen when the potential energy of the initial configurations in the $(2,1)$ mode for shear strains $>4.6 \%$ was minimized. The morphology of the minimum potential energy configuration remained that of the $(2,1)$ mode. Thus, the SWCNT buckled in the $(2,1)$ mode at $4.6 \%$ shear strain. The same holds true for other modes, i.e., if the SWCNT buckles in a mode at a particular strain, then that mode remains unstable for subsequent deformations.

We further substantiate that $4.6 \%$ is indeed the critical strain at which the tube buckles in $(2,1)$ mode. Starting from its configuration in the $(2,1)$ mode, we incrementally deformed the tube in increments of $0.1 \%$ shear strain, and after each incremental shear strain computed its minimum energy configuration. As before for $\gamma<4.6 \%$, the minimum energy configuration of the tube was found to be circular and straight and the potential energy followed the parabolic path $O B$ shown in Fig. 3. However, for $\gamma>4.6 \%$, the minimum energy configuration of the tube remained in the $(2,1)$ mode and the potential energy path bifurcated as shown in Fig. 3 (see filled green diamonds). The morphology of the tube at $\gamma=8 \%$ on the bifurcated energy path is shown in Fig. 2(d). This implies that the tube buckled in the $(2,1)$ mode at $\gamma=4.6 \%$. Using objective MD, Zhang et al. ${ }^{26}$ found a similar bifurcation in the potential energy for infinitely long $(12,12)$ SWCNT deformed in torsion. Their critical value of the shear strain, $6.2 \%$, is about $30 \%$ higher than the present value of $4.6 \%$. This difference is attributed to the method, consideration of clamped boundary conditions and the use of different potentials in the two studies. We note that Annin et $a l .{ }^{31,32}$ used the finite element method and also found bifurcation points in torsional deformations of SWCNTs.

If the ECS of a SWCNT is taken as a shell, then according to the Greenhill buckling condition, ${ }^{45}$ an infinitely long shell under torsion would buckle in $(1,1)$ beam bending mode. This is not captured in Zhang et al's. ${ }^{26}$ symmetryadapted tight-binding objective MD simulations. Since the SWCNT studied here is of finite length, it is not expected to buckle in the $(1,1)$ mode. The results displayed in Fig. 3 evince that the eigenvalue of the $(1,1)$ mode largely remains unaffected for the range of shear strains considered here. Also, we find that the eigenvalue of the torsion mode $(0,1)$ is almost unaffected by shear strains. Furthermore, in our simulations on torsion of $(5,5),(6,6),(8,8),(12,12),(15,15)$, and $(20,20)$ SWCNTs of aspect ratios $\sim 12$, the critical shear strain is found to vary as $(1 / R)^{1.23}$. The exponent 1.23 is between 1.5 obtained by Yakobson et al. $^{2}$ and 0.99 by Zhang et $a l .^{26}$ which could be due to the consideration of finite length SWCNTs with clamped ends. From Fig. 3, we note that the densely populated eigenvalues of $(2, j)$ shell modes at $0 \%$ strain (the relaxed state) start significantly separating with increasing strain. The same feature found for $(3, j)$ modes is not shown for the sake of brevity.

\section{Buckling according to Criterion-1}

With an increase in the shear strain in increments of $0.1 \%$ the potential energy of the SWCNT increases parabolically. Up to the shear strain, $\gamma$, of $11.1 \%$, the minimum energy morphology of the SWCNT is found to be straight and circular as shown in Fig. 2(e). However, in the next incremental deformation, i.e., $\gamma=11.2 \%$, the potential energy of the tube drops sharply and the tube collapses to the morphology with three circumferential waves near the clamped end, as shown in Fig. 2(f). Hence, according to Criterion-1, the tube buckles at $11.2 \%$ strain. Thus, if one adopts Criterion-1, there will be $\sim 143 \%$ error in the critical value of the shear strain for this tube. Furthermore, in order to reconfirm that we have not missed buckling at $4.6 \%$ shear strain, we plot the first derivative of the potential energy with respect to the strain $\gamma$ (i.e., $U^{\prime}$ or the torque generated by reactions at atoms where tangential displacements are prescribed) against the shear strain; it is shown in Fig. 3 by a dashed line. This curve does not have any discontinuity for $\gamma<11.2 \%$, implying that the torque did not suddenly drop for $\gamma<11.2 \%$. This confirms that Criterion-1 significantly overestimates the critical strain.

\section{Discussion}

According to Silvestre's ${ }^{33}$ work, the aspect ratio of the tube under study is $\geq 4.4\left(1-\nu^{2}\right)^{0.25}(R / h)^{0.5}$, where values of 

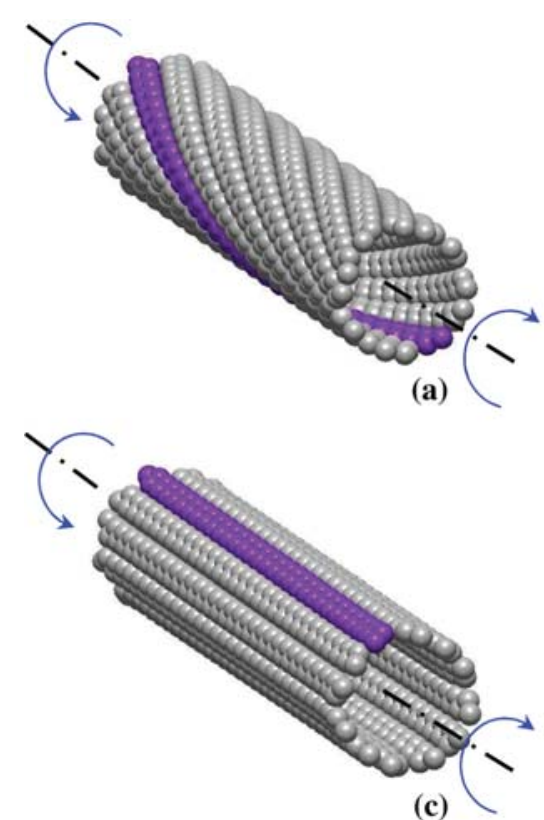

(c)

$\nu$ (Poisson's ratio), $R$ (equilibrated radius of the tube), and $h$ (wall thickness of the tube) are $0.19,7.711 \AA$, and $1.3 \AA$, respectively (see Table III of Ref. 43). Therefore, the critical shear strain for the circumferential wave number $i=2$ can be computed using Donnell's formula, ${ }^{33} \gamma_{c r}=R[(0.544(1+\nu) \div$ $\left.R\left(1-\nu^{2}\right)^{0.75}\right)(h / R)^{1.5}$, where the factor in the square bracket is called the critical angle of twist per unit length. Substituting the values of $\nu, h$ and $R$ for $(12,12)$ SWCNT in this equation yields $\gamma_{c r}=4.62 \%$, which is very close to that obtained by applying Criterion-2. This implies that Donnell's continuum shell model for torsional buckling gives results for this tube that agree well with that derived from Criterion-2 applied to MM simulation findings.

\section{B. Chiral tube}

We now study torsional buckling of an arbitrarily chosen $(8,4)$ chiral SWCNT of aspect ratio $\sim 8$ sheared along and against the direction of chirality.

\section{Buckling according to Criterion-2}

The morphology of $(8,4)$ tube in the reference configuration is shown in Fig. 4(a). The boundary atoms are constrained, and the torsion is applied using the same method as that for the $(12,12)$ tube. A convolving line of purple colored atoms shows the chirality prevalent in the tube, and the blue arrows show positive shear strain in the direction opposite to the chirality. The eigenvalue loci of different modes of vibration and variation in the potential energy for incremental shear strain are shown in Fig. 5. Eigenvalues of the first torsion and the first bending modes are found to increase only slightly with an increase in the shear strain. However, eigenvalues of $(2,1)$ and $(2,2)$ shell modes decrease monotonically for both positive and negative shear strains. According to Criterion-2, the tube buckles in $(2,1)$ mode at $\sim 9.1 \%$ and $\sim 9.5 \%$ shear strain in the negative and the positive directions, respectively.
FIG. 4. Morphologies of $(8,4)$ SWCNT deformed in torsion applied in two different directions. A line of atoms is colored purple to visualize changes in the morphology during the deformation, and the direction of twist is shown by blue arrows. The tube in the equilibrium configuration is shown in (a). The morphology at the collapse when twisted in the direction of chirality is shown in (b). When twisted in the direction opposite of chirality, at $18.4 \%$ shear strain, the helical line of purple colored atoms in (a) becomes parallel to the axis of the tube, shown in (c). The morphology at the collapse when twisted in the direction opposite of chirality is shown in (d). Note that due to the clamped conditions at the end faces, atoms there remain on the undeformed circle.

\section{Buckling according to Criterion-1}

It is evident from the potential energy vs. the shear strain plotted in Fig. 5 that the peak value of the potential energy at the point of collapse during twisting in the direction of chirality $(\sim 5693 \mathrm{kcal} / \mathrm{mol})$ is significantly less than that during twisting in the direction opposite to chirality ( $\sim 8179 \mathrm{kcal} /$ $\mathrm{mol}$ ), and the values of the critical strain in these directions are $18.3 \%$ and $21.6 \%$, respectively. These torsional buckling strains according to Criterion-1 differ by more than $100 \%$ from the values $(\sim 9.1 \%$ and $\sim 9.5 \%)$ found employing Criterion-2.

We have also studied the variation of $U^{\prime}$ with the shear strain and did not notice any discontinuity in it before the points of collapse in either direction; these results are not included here.

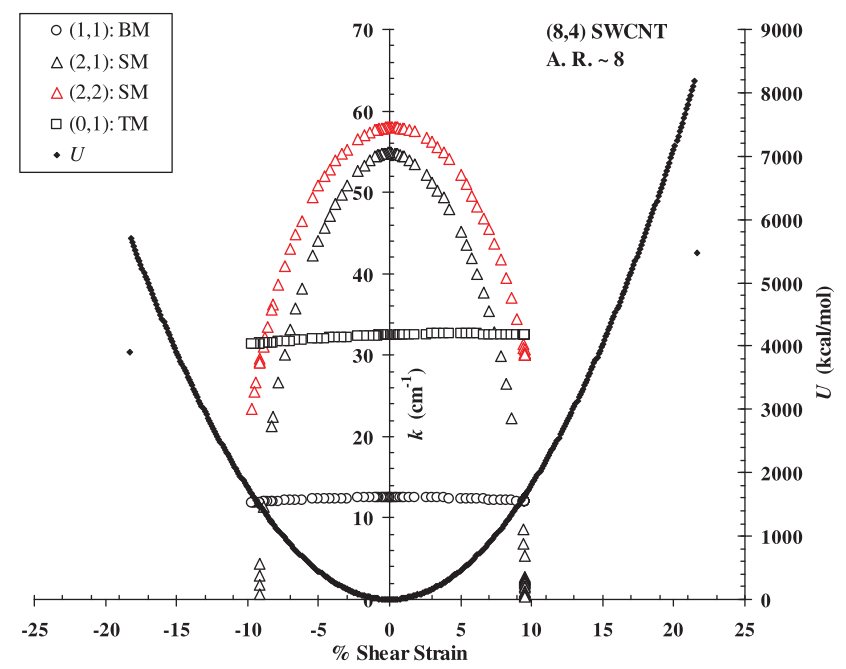

FIG. 5. Variation with shear strain, of the total potential energy $(U)$ and eigenvalues $(k)$ of various modes of vibration (bending $(1,1)$, torsion $(0,1)$, shell $(i, j))$. 


\section{Discussion}

Morphologies of collapsed tubes in the negative and the positive twisting directions are shown in Figs. 4(b) and 4(d). For the positive shear strain, the tube unwinds, and the convolving line formed by the purple colored atoms (cf. Fig. 4(a)) straightens as shown in Fig. 4(c). This morphology corresponds to $18.4 \%$ shear strain. At this strain, the potential energy of the tube is $\sim 6035 \mathrm{kcal} / \mathrm{mol}$. From this state of the tube, it only takes additional $3.2 \%$ shear strain for the tube to collapse. An obvious reason of asymmetry in the potential energy vs. the shear strain plot in Fig. 5 is the chirality of the tube. This asymmetry in values of the critical shear strains sheared in two opposite directions obtained from Criterion-1 has also been reported by $\mathrm{Chang}^{23}$ in his MD simulations of chiral tubes employing the REBO and the L-J potentials. Chang $^{23}$ also presented his findings for the $(8,4)$ tube of aspect ratio 8 . Contrary to our findings, Chang ${ }^{23}$ reported that the critical strain for the tube twisted in the direction of chirality is more than that when it is twisted in the direction opposite to the chirality. Chang ${ }^{23}$ also gave morphologies of $(8,3)$ SWCNT during its torsional deformations. We note that the chiral angle and the aspect ratio of this tube is not that much different from those of the $(8,4)$ tube studied here. Therefore, it is expected that morphologies of these two twisted tubes will also be similar. However, in our studies before collapse the chirality of the $(8,4)$ tube vanishes when it is twisted in a direction opposite to the chirality (cf. Fig. 4(c)) but not in Chang's ${ }^{23}$ results. Another difference is the application of boundary conditions. Unlike in the present simulations, the constrained atoms shown in red color in Fig. 1 of Ref. 23 do not lie on a circle and this is not explained by the author. The difference in results obtained by employing Criterion-1 in the present work and the work of Chang ${ }^{23}$ may be attributed to different MM/MD potentials used and how the clamped boundary conditions are enforced. The asymmetry in the peak value of the potential energy before the onset of collapse in the clockwise and the counter-clockwise twist is not reported in Ref. 23. Finally, we note that the Criterion-2 yields nearly the same value of the critical shear strain in the two directions of twist. Therefore, the direction of the chiral vector is immaterial for using chiral tubes in nano-mechanical systems (NMS) as shafts for transmitting torque.

Interestingly, the asymmetric or the different response of chiral SWCNTs under torsion in two different directions implies inapplicability of continuum shell models presented by Silvestre. ${ }^{33}$ It motivates the development of models for buckling of continuum structures with different elastic moduli in positive and negative twisting directions.

\section{Zigzag tube}

We now report our results for torsional buckling of $(7,0)$ zigzag SWCNT of aspect ratio $\sim 15$.

\section{Buckling according to Criterion-2}

The variations with torsional shear strain of the potential energy and the eigenvalue loci for $(7,0)$ SWCNT are shown in Fig. 6. As for the previously discussed cases, the torsion and bending modes largely remain unaffected with an increase in deformations of the tube. Also, the eigenvalue of the $(2,1)$ shell mode decreases monotonically, and hence, application of Criterion-2 gives the critical buckling strain equal to $\sim 12.33 \%$.

\section{Buckling according to Criterion-1}

As shown in Fig. 6, the potential energy of the $(7,0)$ zigzag SWCNT is found to increase quadratically with strain up to $17 \%$ shear strain without any sharp drop in it. However, there is a discontinuity in the $U^{\prime}$ vs. shear strain graph at $12.7 \%$ shear strain (cf. Fig. 6), which is very close to that found using Criterion-2. In this particular case, we did not deform the tube until it collapsed since we captured a kink in $U^{\prime}$ vs. the shear strain curve that was not found for the $(12,12)$ and the $(8,4)$ SWCNTs.

\section{Discussion}

In order to discuss the applicability of continuum shell models discussed by Silvestre, ${ }^{33}$ we note that the equilibrated radius $(R)$ of the $(7,0)$ SWCNT equaled $2.59 \AA$. Substituting $R=2.59 \AA$ and the equilibrated bond length $=1.34 \AA$ in Eq. (11) of Gupta et al., ${ }^{43}$ the wall thickness $(h)$ of the tube equals $0.69 \AA$. The equation $\nu=-0.0314$ $\ln (R)+0.307$, obtained by the least squares fit to the data of Poisson's ratio, $\nu$, for zigzag tubes computed from the theory of Rayleigh modes and depicted in Fig. 5 of Gupta et al ${ }^{43}$ gives $\nu=\sim 0.3$. The criterion given by Silvestre ${ }^{33}$ implies that the critical shear strain can be computed using Donnell's formula $^{33}$ stated above for $i=2$. The critical shear strain so found equals $10.47 \%$, which is $19 \%$ (12.7 vs. 10.47) less than that found using Criterion-2. This shortfall in the strain may be attributed to the presence of strong van der Waals interactions in MM simulations due to much smaller radius of the $(7,0)$ tube as compared to that of the $(12,12)$ SWCNT, which is not accounted for in the continuum shell model.

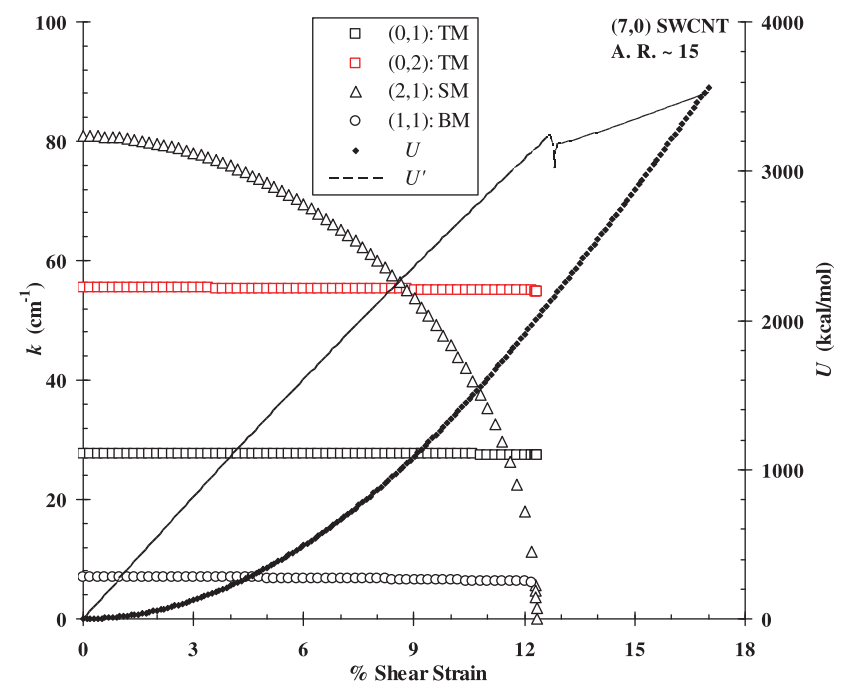

FIG. 6. Variation with the shear strain of the total potential energy $(U)$, its derivative with respect to strain $\left(U^{\prime}\right)$ and eigenvalues $(k)$ of bending, torsion, and shell modes of vibrations. 
In Fig. 6, we have also shown the eigenvalue loci for the second torsion mode. Similar to torsional vibrations of clamped long continuum shafts, for MM simulations, the frequency of the second torsional mode $(0,2)$ is found to be twice that of the first torsional mode $(0,1)$.

\section{AXIAL COMPRESSION OF SWCNTS}

\section{A. Zigzag tube}

Here, we report our results for axial buckling of $(7,0)$ zigzag SWCNT of aspect ratio $\sim 15$.

\section{Buckling according to Criterion-1}

We use the procedure outlined in Section II to analyze buckling of the $(7,0)$ tube. The variation in the potential energy of the SWCNT and the eigenvalue loci of various modes at different values of the axial strain are shown in Fig. 7. The potential energy of the structure suddenly decreases at $7.18 \%$ compressive axial strain with the tube morphology shown in Fig. 7. At the tube's mid-point, the collapsed crosssection has two circumferential waves. Thus, according to Criterion-1, the buckling strain is $7.18 \%$. In order to ensure that it is the lowest buckling strain for Criterion-1, we have plotted $U^{\prime}$ versus the axial strain in Fig. 7. This plot does not show any discontinuity for strains $<7.18 \%$, and hence, according to Criterion-1, the value of $\varepsilon_{c r}$ is $7.18 \%$.

\section{Buckling according to Criterion-2}

The examination of eigenvalue loci reveals that the tube buckles in the first and the second bending modes at $2.2 \%$ and $4.3 \%$ compressive axial strain, respectively. We note that the critical buckling strain computed using Criterion-1 is nearly three times that found using Criterion-2. Once the first bending mode $(1,1)$ becomes unstable, i.e., the lowest eigenvalue $=0$ for this mode, it remains so for all subsequent incremental compressive strains. The same holds true for the second bending mode $(1,2)$. In order to avoid cluttering data on the $x$-axis, we have shown zero eigenvalues of bending modes only at a few points. The eigenvalues of the bending modes in Fig. 7 after the tube has become unstable coincide with each other. The eigenvalue of the third bending mode $(1,3)$ is also found to decrease monotonically with an increase in the axial strain. The cross-section of the midsection of buckled tubes corresponding to the first three bending modes obtained from Criterion-2 (cf. Fig. 7) is circular. The eigenvalue loci of the first torsion mode, shown in Fig. 7, are found to vary only slightly with an increase in the compressive axial strain. We have also studied the effect of the tensile axial strain on the potential energy and the eigenvalue loci up to $0.6 \%$ strain for the sake of completeness. With increasing tensile axial strain, the potential energy is found to vary quadratically and the eigenvalues are found to increase monotonically (cf. Fig. 7). The increase in the magnitude of eigenvalues with an increase in the axial tensile strain is similar to what occurs in strings and beams. ${ }^{44}$ The increase in the eigenvalues and in the potential energy is expected to cease at the tensile axial strain at which bonds begin to break.

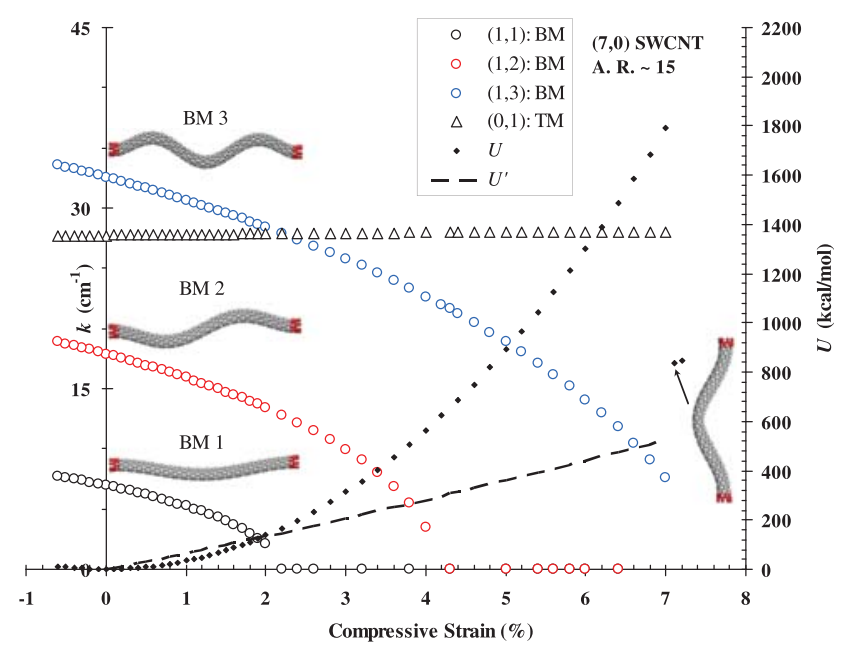

FIG. 7. Variation with the axial compressive strain of the total potential energy $(U)$ and its derivative with respect to strain $\left(U^{\prime}\right)$, and eigenvalues $(k)$ of various modes of vibration (bending (BM) and torsion (TM)). The first three bending modes are shown in the figure. The red atoms are held fixed. The morphology at $7.1 \%$ compressive axial strain is also shown.

\section{Discussion}

One expects that the Euler column buckling theory ${ }^{45}$ should predict the buckling of slender tubes (aspect ratio $>10$ ) and the lowest critical buckling strain $\varepsilon_{c r}=2(\pi / L)^{2}\left(R^{2}+h^{2} / 4\right)$. Substituting in this equation, the values of $R$ and $h$ computed in the Section III C 3 for the $(7,0)$ tube yield $\varepsilon_{c r}=2.3 \%$, which agree well with those found using Criterion-2.

\section{B. Armchair tube}

We now report our results for axial buckling of $(7,7)$ SWCNT of aspect ratio $\sim 6.4$ studied by Yakobson et al., ${ }^{2}$ under compression.

\section{Buckling according to Criterion-1}

For the above mentioned tube, we see from results exhibited in Fig. 8 that Criterion-1 gives $\varepsilon_{c r}=10.1 \%$ where the potential energy suddenly drops. This is confirmed by the absence of any discontinuity in the graph of $U^{\prime}$ vs. $\varepsilon_{c}$ up to $10.1 \%$ axial compressive strain (cf. Fig. 8). The morphology of the collapsed tube at $10.1 \%$ axial strain is also shown in Fig. 8. Unlike for tubes with large aspect ratio, for the $(7,0)$ SWCNT of aspect ratio $\sim 6.4$, the deformed configuration is asymmetric, and the crimped zone is closer to the end which was incrementally moved. This morphology is quite different from that obtained by Yakobson et al. ${ }^{2}$ at the first instance of the drop in the potential energy at 5\% axial strain; however, it resembles the morphology reported by Yakobson et al. ${ }^{2}$ at $13 \%$ axial strain.

\section{Buckling according to Criterion-2}

As shown in Fig. 8, eigenvalues of the first bending mode $(1,1)$ and of the shell modes $(2,1),(2,2),(2,3)$, and $(2,4)$ become zero almost at the same value of the compressive axial strain $=\sim 9.12 \%$, irrespective of their much different eigenvalues in the relaxed state, i.e., $0 \%$ strain. The eigenvalue loci of the shell modes $(2,1),(2,2)$, and $(2,3)$ are 


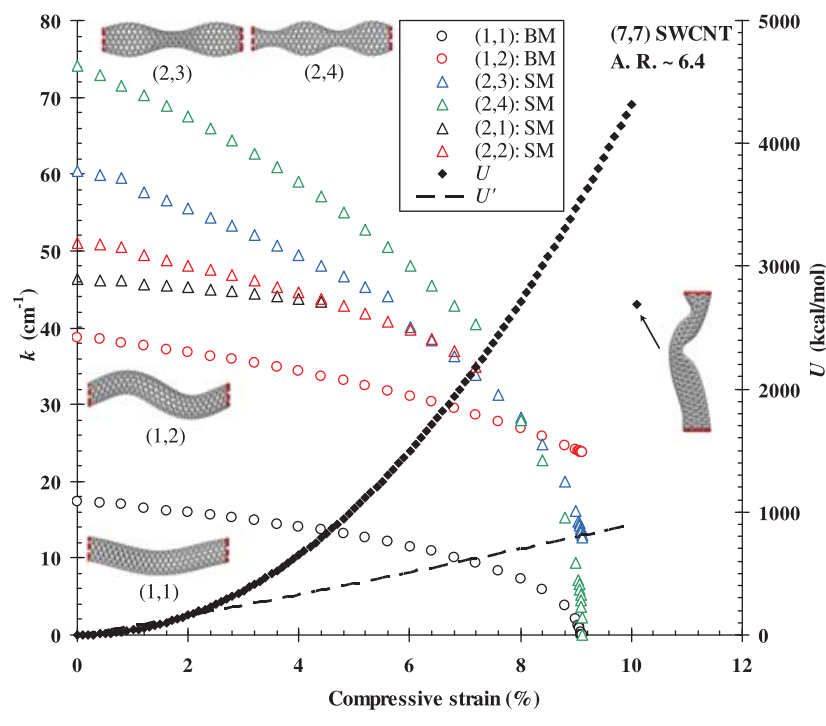

FIG. 8. Variation with the axial compressive strain of the total potential energy $(U)$, its derivative with respect to strain $\left(U^{\prime}\right)$, and eigenvalues $(k)$ of bending $(\mathrm{BM})$ and shell $(\mathrm{SM})$ modes. Four vibration modes and the tube morphology at $10.1 \%$ compressive axial strain are also shown. The red atoms are held fixed.

found to coalesce after $6 \%$ axial compression and it became impossible to distinguish these modes from each other in the simulations. Similarly, after $8 \%$ axial compression, the eigenvalue loci of the $(2,4)$ mode coalesces with that of the other three, and the eigenvalues of all four modes become almost zero at $\sim 9.12 \%$ axial strain, thereby indicating multiple bifurcation branches; this feature of shell modes is discussed in the next paragraph. For this short tube, Criterion-1 over-predicts the value of the critical strain by about $11 \%$ (10.1\% vs. $9.12 \%)$ as compared to that given by Criterion-2.

\section{Discussion}

The Euler column buckling formula used above to compute $\varepsilon_{c r}$ for the $(7,0)$ SWCNT cannot be directly applied to find $\varepsilon_{c r}$ for the $(7,7)$ tube because its aspect ratio is $<10$. However, the shear corrected formula, ${ }^{45} \varepsilon_{S c}=\varepsilon_{c r} /\left[1+(1+\nu) \kappa \varepsilon_{c r}\right]$, in which the shear correction factor $\kappa$ depends on the cross section of the structure, is applicable. The values of $\nu, R$, and $h$ for the $(7,7)$ SWCNT from Table III of Ref. 43 are $0.16,4.5 \AA$, and $1.09 \AA$, respectively, and $\kappa=1.82$ for the circular crosssection calculated using the one proposed by Hutchinson. ${ }^{46}$ Thus, the first critical buckling strain $\varepsilon_{S c}=9.82 \%$, which is $\sim 7 \%(9.82 \%$ vs. $9.12 \%)$ higher than that found from Criterion-2. Furthermore, the critical buckling strain for the formation of two circumferential waves $(i=2)$ using the formula $^{45} \varepsilon_{c r(2, j)}=(3 / 5)(h / R)\left(3\left(1-\nu^{2}\right)\right)^{-1 / 2}$ equals 0.085, which is 0.0062 less than that obtained from Criterion-2. The formula for $\varepsilon_{c r(2, j)}$ is independent of the tube length; thus, the value of $\varepsilon_{c r(2, j)}$ is independent of the tube aspect ratio.

From Fig. 3 of Silvestre, ${ }^{47}$ we find that the critical buckling strain of the shell modes $(2, j), j=1,2, \ldots, 5$, is the same for $\sim 20<L<100$. In order to verify this, we studied axial buckling of the $(7,7)$ SWCNT of aspect ratio $=3.65$ ( $L \sim 33 \AA$ ) using MM simulations. The variation in the potential energy, and the eigenvalue loci of the first bending and the first two shell modes are shown in Fig. 9. It is evident from the eigenvalue loci of the $(2,1)$ and the $(2,2)$ modes that the critical strain equals $\sim 9.9 \%$, which is very close to that found for the tube of aspect ratio $\sim 6.4$. Thus, we find that for the $(7,7)$ tube, critical buckling strains from the MM simulations agree well with those from the continuum shell theory. However, we conclude from results depicted in Fig. 9 that Criterion-1 overestimates the critical buckling strain. For the shorter tube, it is expected that the buckling strain for the $(1,1)$ flexural mode will be higher than that for the longer tube. For the tube of aspect ratio $\sim 6.4$, matching of the critical strains for the $(1,1)$ bending mode and the $(2, j)$ shell modes was merely a coincidence.

\section{EFFECT OF ASPECT RATIO ON CRITICAL STRAINS}

Critical buckling strains according to the two criteria found for SWCNTs of different aspect ratios under torsional and axial loading are listed in Tables I and II. It is clear that the two criteria give different buckling strains for all the tubes studied, implying that there is no correlation between the buckling strains according to the two criteria and the tube's chirality and aspect ratio.

\section{AN EXPLANATION FOR THE DIFFERENCES IN CRITICAL STRAINS FROM TWO CRITERIA}

In an attempt to investigate if local instabilities at the atomic level trigger the global instability characterized by the buckling instability, for each increment in torsional loading of the $(12,12)$ SWCNT, we have computed the total potential energy of each atom as well as the total potential energy of the entire tube. The fringe plots of the local energy exhibited in Fig. 10 reveal that the local energy distribution is uniform at shear strains of $4.6 \%$ and $11.1 \%$, the cross-sections remain circular, and the total potential energies of the SWCNT equal 1858 and $10586 \mathrm{kcal} / \mathrm{mol}$, respectively, cf. Figs. 10(a) and 10(b). Note that according to Criterion-2, the tube buckles at $4.6 \%$ shear strain. The fringe

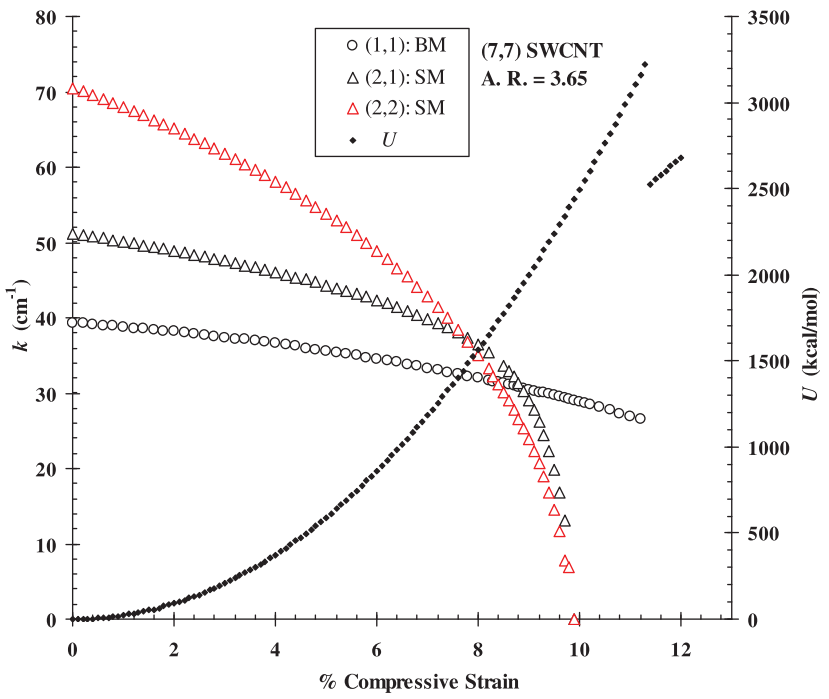

FIG. 9. Variation with the axial compressive strain of the total potential energy $(U)$, and eigenvalues $(k)$ of bending $(\mathrm{BM})$ and shell (SM) modes. 
TABLE I. Axial buckling: Critical compressive strain from Criterion- 1 and Criterion-2.

\begin{tabular}{lccc}
\hline \hline SWCNT & $\begin{array}{c}\text { Aspect ratio } \\
\text { (approximate) }\end{array}$ & $\begin{array}{c}\text { Critical strain } \\
\text { (\%) Criterion-1 }\end{array}$ & $\begin{array}{c}\text { Critical strain } \\
\text { (\%) Criterion-2 }\end{array}$ \\
\hline$(10,10)$ & 15 & 7.60 & 1.95 \\
$(7,7)$ & 15 & 7.40 & 2.21 \\
$(7,0)$ & 15 & 7.18 & 2.20 \\
$(7,7)$ & 3.65 & 11.30 & 9.90 \\
$(7,7)$ & 6.4 & 10.00 & 9.12 \\
\hline \hline
\end{tabular}

TABLE II. Torsion buckling: Critical shear strain from Criterion-1 and Criterion-2.

\begin{tabular}{lccc}
\hline \hline SWCNT & $\begin{array}{c}\text { Aspect ratio } \\
\text { (approximate) }\end{array}$ & $\begin{array}{c}\text { Critical strain (\%) } \\
\text { Criterion-1 }\end{array}$ & $\begin{array}{c}\text { Critical strain (\%) } \\
\text { Criterion-2 }\end{array}$ \\
\hline$(8,4)$ & 8 & 21.5 & 9.54 \\
$(7,0)$ & 15 & $>17$ & 12.33 \\
$(12,12)$ & 12 & 11.1 & 4.60 \\
$(7,7)$ & 15 & 9 & 8.3 \\
$(5,5)$ & 12 & 15.00 & 14.20 \\
$(6,6)$ & 12 & 10.4 & 9.60 \\
$(8,8)$ & 12 & $>12$ & 7.2 \\
$(12,12)$ & 12 & 11.2 & 4.6 \\
$(15,15)$ & 12 & 3.9 & 3.4 \\
$(20,20)$ & 12 & $>4.0$ & 2.4 \\
$(7,7)$ & 4 & 12.4 & 10.2 \\
$(7,7)$ & 6.4 & 10 & 9 \\
\hline \hline
\end{tabular}

(a)

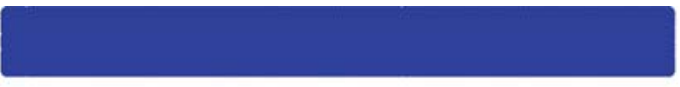

(b)

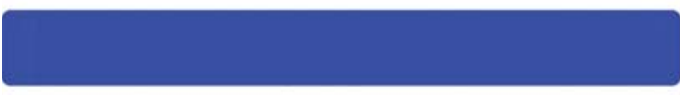

(c)

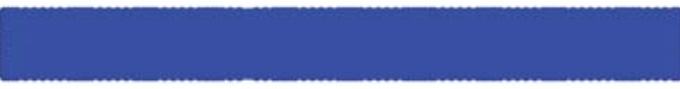

(d)

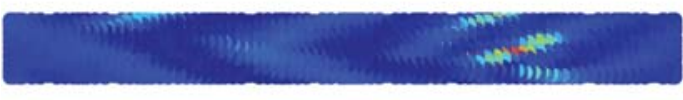

(e)

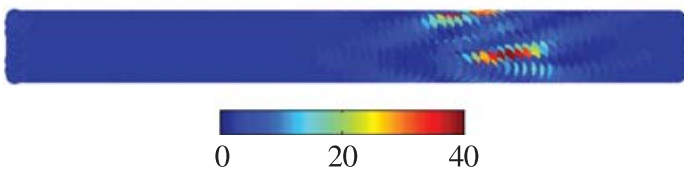

FIG. 10. Fringe plots of the total potential energy of atoms of the $(12,12)$ SWCNT at (a) $4.6 \%$, (b) $11.1 \%$, (c) $11.15 \%$, (d) $11.16 \%$, and (e) $11.2 \%$ shear strains. The unit of color bar is kcal/atom.

plots of local potential energy exhibited in Figs. 10(c)-10(e) at shear strains of $11.15 \%, 11.16 \%$, and $11.2 \%$, respectively, show that at $11.16 \%$ shear strain there is a redistribution of atomic energies and the potential energy of a few atoms is much larger than that of the remaining atoms. Furthermore, bands of different energy levels appear in Figs. 10(d) and 10 (e) at torsional strains of $11.16 \%$ and $11.2 \%$, respectively. Thus, we conclude that at $11.2 \%$ shear strain, the collapse of the SWCNT is due to local activity whereas the vanishing of the non-trivial eigenvalue of the Hessian or of the stiffness matrix corresponds to the onset of the global instability. In a future study, we will explore if the Criterion-1 always corresponds to the onset of a local instability in a SWCNT.

\section{CONCLUSIONS}

We have used MM simulations to study buckling of SWCNTs deformed in either axial compression or torsion. Buckling under axial compression is studied only for armchair and zigzag SWCNTs, but that under torsion is also investigated for a chiral SWCNT. Different values of the critical buckling strain are found from the following two criteria, namely, (i) a sudden drop in the potential energy of the system, and (ii) vanishing of otherwise non-trivial eigenvalue of the mass weighted Hessian of the system. For torsional deformations, the critical buckling strain according to Criterion-1 is more than twice of that obtained from Criterion-2. A similar difference in the two critical buckling strains is found for axial buckling of SWCNTs of the aspect ratio $>6$. The critical buckling strains predicted from the continuum column and shell theories are very close to those obtained from Criterion-2. Thus, the widely used buckling criterion based on the sudden drop in the potential energy of the system during the deformation process gives results quite different from those derived by using the criterion based on the vanishing of the fundamental eigenvalue of the mass weighted Hessian of the system. For torsional deformation of the $(12,12)$ SWCNT, the critical shear strain from Criterion-1 corresponded to the onset of local instabilities at different atomic locations as indicated by a sudden change in their potential energies.

\section{ACKNOWLEDGMENTS}

R.C.B. gratefully acknowledges the partial support of the work from the Office of Naval Research Grant No. N00014-16-1-2309 to Virginia Polytechnic Institute and State University with Dr. Y. D. S. Rajapakse as the program manager.

${ }^{1}$ L. D. Landau, L. P. Pitaevskii, A. M. Kosevich, and E. M. Lifshitz, Theory of Elasticity, 3rd ed. (Butterworth-Heinemann, Oxford, 1986).

${ }^{2}$ B. I. Yakobson, C. J. Brabec, and J. Bernholc, Phys. Rev. Lett. 76, 2511 (1996).

${ }^{3}$ D. Srivastava, M. Menon, and K. Cho, Phys. Rev. Lett. 83, 2973 (1999).

${ }^{4}$ K. Liew, C. Wong, X. He, M. Tan, and S. Meguid, Phys. Rev. B 69, 115429 (2004).

${ }^{5}$ O. Lourie, D. M. Cox, and H. D. Wagner, Phys. Rev. Lett. 81, 1638 (1998).

${ }^{6}$ A. Sears and R. C. Batra, Phys. Rev. B: Condens. Matter Mater. Phys. 69, 235406 (2004).

${ }^{7}$ G. Cao and X. Chen, Phys. Rev. B 74, 165422 (2006).

${ }^{8}$ C.-L. Zhang and H.-S. Shen, Carbon 44, 2608 (2006).

${ }^{9}$ C. L. Zhang and H. S. Shen, Phys. Rev. B: Condens. Matter Mater. Phys. 75, 45408 (2007).

${ }^{10}$ Q. Wang, S. T. Quek, and V. K. Varadan, Phys. Lett. A 367, 135 (2007).

${ }^{11}$ H. Xin, Q. Han, and X. H. Yao, Carbon 45, 2486 (2007).

${ }^{12}$ Y. Y. Zhang, V. B. C. Tan, and C. M. Wang, Carbon 45, 514 (2007).

${ }^{13}$ Q. Wang, K. M. Liew, and W. H. Duan, Carbon 46, 285 (2008).

${ }^{14}$ Y. Y. Zhang, Y. Xiang, and C. M. Wang, J. Appl. Phys. 106, 113503 (2009).

${ }^{15}$ V. Vijayaraghavan and C. H. Wong, Comput. Mater. Sci. 71, 184 (2013).

${ }^{16}$ Y. D. Kuang, X. Q. He, C. Y. Chen, and G. Q. Li, Carbon 47, 279 (2009). 
${ }^{17}$ A. Garg and S. B. Sinnott, Chem. Phys. Lett. 295, 273 (1998).

${ }^{18}$ C. H. Wong and V. Vijayaraghavan, Phys. Lett. A 378, 570 (2014).

${ }^{19}$ G. Cao, X. Chen, and J. Kysar, Phys. Rev. B 72, 195412 (2005).

${ }^{20}$ T. Chang, G. Li, and X. Guo, Carbon 43, 287 (2005).

${ }^{21}$ S. Yeak, T. Ng, and K. Liew, Phys. Rev. B 72, 165401 (2005).

${ }^{22}$ A. Garg, J. Han, and S. B. Sinnott, Phys. Rev. Lett. 81, 2260 (1998).

${ }^{23}$ T. Chang, Appl. Phys. Lett. 90, 201910 (2007).

${ }^{24}$ Y. R. Jeng, P. C. Tsai, and T. H. Fang, Appl. Phys. Lett. 90, 161913 (2007).

${ }^{25}$ B. W. Jeong, J. K. Lim, and S. B. Sinnott, J. Appl. Phys. 101, 84309 (2007).

${ }^{26}$ D. B. Zhang, R. D. James, and T. Dumitrica, Phys. Rev. B: Condens. Matter Mater. Phys. 80, 115418 (2009).

${ }^{27}$ F. Khademolhosseini, R. K. N. D. Rajapakse, and A. Nojeh, Comput. Mater. Sci. 48, 736 (2010).

${ }^{28}$ R. H. Poelma, H. Sadeghian, S. Koh, and G. Q. Zhang, Microelectron. Reliab. 52, 1279 (2012).

${ }^{29}$ M. Eftekhari, S. Mohammadi, and A. R. Khoei, Comput. Mater. Sci. 79, 736 (2013).

${ }^{30}$ S. C. Chowdhury, B. Z. Haque, and J. W. Gillespie, Comput. Mater. Sci. 82, 503 (2014).

${ }^{31}$ B. D. Annin, S. N. Korobeynikov, and A. V. Babichev, J. Appl. Ind. Math. 3, 318 (2009).
${ }^{32}$ B. D. Annin, V. V. Alekhin, A. V. Babichev, and S. N. Korobeynikov, Mech. Solids 47, 544 (2012).

${ }^{33}$ N. Silvestre, Eur. J. Mech., A 32, 103 (2012).

${ }^{34}$ Y. Kinoshita, M. Kawachi, T. Matsuura, and N. Ohno, Phys. E 54, 308 (2013).

${ }^{35} \mathrm{H}$. Shima, Materials 5, 47 (2012).

${ }^{36}$ N. L. Allinger, Y. H. Yuh, and J. H. Lii, J. Am. Chem. Soc. 111, 8551 (1989).

${ }^{37} \mathrm{~J}$. W. Ponder, User's Guide, TINKER - Software Tools for Molecular Design, 2015.

${ }^{38}$ S. S. Gupta and R. C. Batra, Comput. Mater. Sci. 43, 715 (2008).

${ }^{39}$ A. Rao, E. Richter, S. Bandow, and B. Chase, Science 275, 187 (1997).

${ }^{40}$ S. S. Gupta and R. C. Batra, J. Comput. Theor. Nanosci. 7, 2151 (2010).

${ }^{41}$ C. Lee, X. Wei, J. W. Kysar, and J. Hone, Science 321, 385 (2008).

${ }^{42}$ P. H. Shah and R. C. Batra, Comput. Mater. Sci. 83, 349 (2014).

${ }^{43}$ S. S. Gupta, F. G. Bosco, and R. C. Batra, Comput. Mater. Sci. 47, 1049 (2010).

${ }^{44} \mathrm{P}$. Hagedorn and A. DasGupta, Vibrations and Waves in Continuous Mechanical Systems, 1st ed. (Wiley, 2007).

${ }^{45}$ S. P. Timoshenko and J. M. Gere, Theory of Elastic Stability, 1st ed. (Dover Publications, Inc., New York, 2009).

${ }^{46}$ J. R. Hutchinson, J. Appl. Mech. 68, 87 (2000).

${ }^{47}$ N. Silvestre, Int. J. Solids Struct. 45, 4902 (2008).

${ }^{48}$ T. Dumitrica and R. D. James, J. Mech. Phys. Solids 55, 2206 (2007). 